\title{
Ursolic acid ameliorates adipose tissue insulin resistance in aged rats via activating the Akt-glucose transporter 4 signaling pathway and inhibiting inflammation
}

\author{
TONG-ZHUANG WANG ${ }^{1}$, GUO-WEI ZUO ${ }^{2}$, LING YAO $^{1}$, CHUN-LIN YUAN $^{1}$, HAI-FEI LI ${ }^{3}$, YING LAI $^{4}$, \\ ZHI-WEI CHEN $^{1}$, JUN ZHANG ${ }^{3}$, YA-QIAN JIN ${ }^{3}$, JOHJI YAMAHARA ${ }^{5}$ and JIAN-WEI WANG ${ }^{1}$ \\ ${ }^{1}$ Chongqing Key Laboratory of Traditional Chinese Medicine for Prevention and Cure of Metabolic Diseases, College of \\ Traditional Chinese Medicine; ${ }^{2}$ Laboratory of Medical Tests; ${ }^{3}$ Faculty of Basic Medical Sciences; \\ ${ }^{4}$ Institute of Life Sciences, Chongqing Medical University, Chongqing 400016, P.R. China; \\ ${ }^{5}$ Pharmafood Institute, Kyoto 602-8136, Japan
}

Received September 9, 2019; Accepted April 7, 2021

DOI: $10.3892 /$ etm.2021.10901

\begin{abstract}
Ageing often results in insulin resistance (IR) and chronic inflammation, and adipose is one of the tissues in which inflammation and IR occur earliest during this process. The present study investigated the effect and underlying mechanisms of ursolic acid (UA) on adipose IR and inflammation in ageing rats. Specific pathogen-free male Sprague-Dawley rats were randomly divided into 4 groups: i) Young normal (young); ii) untreated ageing (aged); and groups supplemented with UA either iii) low-UA $10 \mathrm{mg} / \mathrm{kg}$ (UA-L) or iv) high-50 mg/kg (UA-H). Animals in the UA-treated groups received 10 or $50 \mathrm{mg} / \mathrm{kg}$ UA (suspended in 5\% Gum Arabic solution). The rats in the corresponding aged group and young groups received vehicle (5\% Gum Arabic) alone. All rats were intragastrically treated once daily by oral gavage for 7 weeks. The day before the experiment terminated, overnight fasting blood $(\sim 700 \mu 1)$ was collected and plasma was prepared to measure biochemical indicators; western blotting was performed to analyze the expression of insulin signaling proteins [(insulin receptor substrate 1 (IRS-1), phosphorylated (p)-IRS-1, PI3K, glucose transporter 4 (GLUT4), Akt and $\mathrm{p}-\mathrm{Akt})]$ and inflammatory factors $(\mathrm{NF}-\kappa \mathrm{B}, \mathrm{IL}-6$ and IL-1 $\beta$ ) in the epididymis white adipose tissue (eWAT). The results revealed that treatment with UA-H decreased eWAT weight, the ratio of eWAT weight/body weight, fasted insulin and triglyceride levels, the homeostasis model assessment of insulin resistance and adipose tissue insulin resistance index in ageing
\end{abstract}

Correspondence to: Professor Jian-Wei Wang, Chongqing Key Laboratory of Traditional Chinese Medicine for Prevention and Cure of Metabolic Diseases, College of Traditional Chinese Medicine, Chongqing Medical University, 1 Medical School Road, Chongqing 400016, P.R. China

E-mail: wangjianwei@cqmu.edu.cn

Key words: ursolic acid, ageing, insulin resistance, inflammation rats, indicating the amelioration of systemic and adipose tissue IR, compared with the aged group. Mechanistically, UA-H administration upregulated p-protein kinase $\mathrm{B}$, the ratio of p-Akt to protein kinase B and total and cellular membrane GLUT4 protein levels in eWAT of ageing rats. Conversely, UA inhibited the increase in $\mathrm{NF}-\kappa \mathrm{B}$ expression and proinflammatory cytokines IL- 6 and IL-1 $\beta$. However, these alterations were not observed in the rats of the aged group. Taken together, the findings of the present study indicated that UA may ameliorate adipose IR, which is associated with activation of the Akt-GLUT4 signaling pathway and inhibition of inflammation in ageing rats. These data provide a basis for the development of effective and safe drugs or functional substances, such as $\mathrm{UA}$, for the prevention and treatment of metabolic diseases.

\section{Introduction}

The ageing of the population is a global phenomenon. The World Health Organization (WHO) reported that the number of people over the age of 65 will exceed the number of children under the age of 5 by 2020 (1). Ageing is a major risk factor for the development of metabolic disorders, such as insulin resistance (IR) and obesity (2,3).

IR is a decrease in the ability of insulin to maintain normal blood glucose levels, meaning that a certain concentration of insulin will not produce the desired physiological effect, and the response of tissues, such as liver tissue, adipose tissue and skeletal muscle, to insulin decreases. Glucose toxicity is a significant cause of IR (4). In the IR state, the uptake and utilization of glucose by peripheral tissues, including liver tissue, adipose tissue and skeletal muscle) is significantly decreased $(5,6)$. Adipose tissue is considered to be one of the major peripheral tissues that develops IR $(7,8)$. In particular, white adipose tissue serves an active role in IR by secreting inflammatory factors, including tumor necrosis factor- $\alpha$ (TNF- $\alpha$ ), interleukin (IL)-6 and monocyte chemotactic protein (MCP)1 (9). Pro-inflammatory cytokines, particularly TNF- $\alpha$, could enhance the serine phosphorylation of insulin receptor substrate-1 (IRS-1), which induced insulin resistance (10). 
The PI3K/Akt pathway serves a crucial role in insulin signaling and insulin-mediated glucose metabolism pathways (11-13). In addition, the PI3K/Akt pathway is the primary signal transduction pathway downstream of the insulin receptor, which is closely associated with the development of diabetes and decreased insulin sensitivity (14). In adipocytes, activated PI3K and Akt promote translocation and fusion of glucose transporter 4 (GLUT4)-containing vesicles to the membrane, increasing GLUT4 transporter protein and glucose uptake at the membrane surface $(15,16)$. Hence, impairment of $\mathrm{PI} 3 \mathrm{~K} / \mathrm{Akt}$ signaling pathways may be the underlying mechanism associated with IR.

Ursolic acid (UA) is a triterpenoid compound widely found in natural plants, such as crataegus pinnatifida Bunge and fructus mume. UA has gained attention due to its varied effects including anti-inflammatory, antioxidant, anticarcinogenic, antimicrobial, antiviral and hepatoprotective actions $(17,18)$. Previous studies have confirmed that UA can increase insulin sensitivity, improve glucose and lipid metabolism disorders, and hence alleviate the role of metabolic syndrome in high-fat diet-induced non-alcoholic fatty liver disease rats $(19,20)$. Our previous study has also demonstrated that a mixture of malus domestica and rosmarinus officinalis extract (rich in UA) improved IR induced by high fructose in rats (21). However, to the best of our knowledge there has been no study yet that has investigated the effects and underlying mechanisms of UA on age-associated IR.

In the present study, the effect of UA on adipose tissue IR was investigated in ageing rats. To explore its potential mechanism, IRS-1/PI3K/Akt signaling pathway and inflammatory factors $(\mathrm{NF}-\kappa \mathrm{B}, \mathrm{IL}-6, \mathrm{IL}-1 \beta)$ in epididymis white adipose tissue (eWAT) were examined. The results provided a molecular pharmacological basis for UA to prevent and treat metabolic diseases.

\section{Materials and methods}

Animals. Male young (3-month; body weight, 210 $\pm 20 \mathrm{~g}$ ) and old (22-month; body weight, 550 \pm 20 g) Sprague-Dawley rats [Laboratory animal certificate number, SCXK (Chongqing) 2018-0003] were supplied by the Laboratory Animal Centre of Chongqing Medical University (Chongqing, China). The rats were housed in a temperature-controlled facility at $21 \pm 1^{\circ} \mathrm{C}$ under a 12/12-h light/dark cycle and a relative humidity of $55 \pm 5 \%$ in a specific pathogen-free facility with free access to water and food. All animal experiments were performed in accordance with the institutional and national guidelines and regulations, and approved by the Animal Ethics Committee of Chongqing Medical University.

Reagents. The following reagents were used in the present study. Ursolic acid (donated by Pharmafood Institute; Kyoto, Japan), arabic gum (cat. no. MC0124B1013J; Shanghai Shenggong Biology Engineering Technology Service, Ltd.), isoflurane (cat. no. 217180801; Shenzhen Rui Wode Life Technology Co., Ltd.), Triglyceride Reagent kit (TG; cat. no. 2017040017; Zhejiang Dongpu Diagnostic Products Co., Ltd.), Glucose Reagent kit (cat. no. 20171004147; Shanghai Rongsheng Biological Pharmaceutical Co., Ltd.), non-esterified fatty acid (NEFA) Reagent kit (cat. no. AR083; Fujifilm
Wako Pure Chemical Corporation), Insulin Reagent kit (cat. no. 13SERI049; Morinaga Institute of Biological Science, Inc.) and RIPA buffer (Strong; cat. no. P0013B; Beyotime Institute of Biotechnology).

Experimental protocol. Following 1 week of adaptable feeding, 25 ageing model rats were divided initially into 3 groups: i) Untreated ageing group (aged; $n=9)$; ii) group supplemented with low UA $10 \mathrm{mg} / \mathrm{kg}$ (UA-L; n=8); and iii) high $50 \mathrm{mg} / \mathrm{kg}$ (UA-H; n=8) (21-23). A total of 8 young rats were randomly selected as the young untreated group (young). Animals in the UA-treated groups received UA suspended in 5\% arabic gum solution and administered via oral gavage once daily for 7 weeks. The rats in the corresponding untreated aged and young groups received vehicle (5\% arabic gum) alone for 7 weeks. All rats had free access to a standard diet. The consumed goods and body weight of the animals was measured every 2 days and the behavior of the rats was monitored. The study was scheduled for 7 weeks. The day before the experiment was terminated, the rats were deprived of food, but still had free access to water overnight (14 h). Subsequently, all the animals were weighed, anesthetized with inhaled isoflurane at a concentration of 3-4\% and sacrificed via prompt cervical dislocation. Death was confirmed in the animals by the disappearance of breathing and heartbeat. Subsequently, eWAT were collected and weighed and immediately frozen in liquid nitrogen and stored at $-80^{\circ} \mathrm{C}$ until further use.

Biochemical determination. The day before the experiment was terminated, all rats were deprived of food, but still had free access to water for $14 \mathrm{~h}$. Blood samples $(\sim 700 \mu \mathrm{l})$ were collected via retro-orbital venous puncture under isoflurane anesthesia, then plasma was extracted by centrifugation at $1,200 \mathrm{x} \mathrm{g}$ for $15 \mathrm{~min}$ at $4^{\circ} \mathrm{C}$ for determination of concentrations of glucose. All the kits were used according to the manufacturer's instructions.

Detection of homeostasis model assessment of insulin resistance (HOMA-IR) and adipose tissue insulin resistance (Adipo-IR) indices. The following formulae were used to determine HOMA-IR and the Adipo-IR indices: HOMA-IR index $=[$ fasted insulin level $(\mathrm{mIU} / \mathrm{ml}) \times$ fasted glucose level $(\mathrm{mM})] / 22.5$ (24); Adipo-IR=fasted insulin (mmol/l) $\mathrm{x}$ fasted NEFA (pmol/1) (25-29).

Western blotting. Total, plasma and membrane protein was prepared from eWAT using the Minute ${ }^{\mathrm{TM}}$ Plasma Membrane Protein Isolation kit (cat. no. SM-005 Minute ${ }^{\mathrm{TM}}$ Plasma Membrane Protein Isolation kit (cat. no. SM-005; Invent Biotechnologies, Inc.) according to the manufacturer's instructions. The epididymal adipose tissue was homogenized with RIPA buffer supplemented with a 1:100 dilution of protease and phosphatase inhibitors (PMSF) (cat. no. P8340 and cat. no. P5726, respectively; Sigma-Aldrich; Merck KGaA). The protein concentration was determined using a bicinchoninic acid (BCA) protein concentration assay kit (Enhanced; cat. no. P0010S; Beyotime Institute of Biotechnology). Proteins $(25 \mu \mathrm{g})$ were separated via $8 \%$ SDS-PAGE under reducing conditions and transferred to PVDF membranes (GE Healthcare). Membranes were 

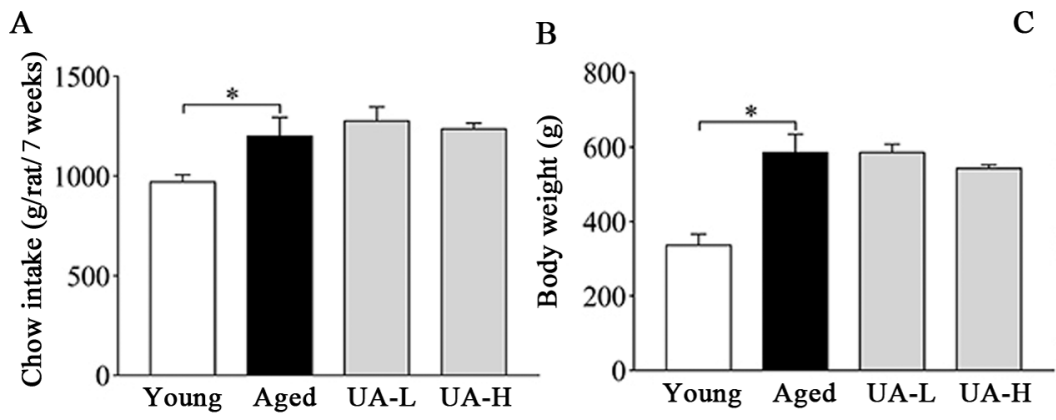

C

D

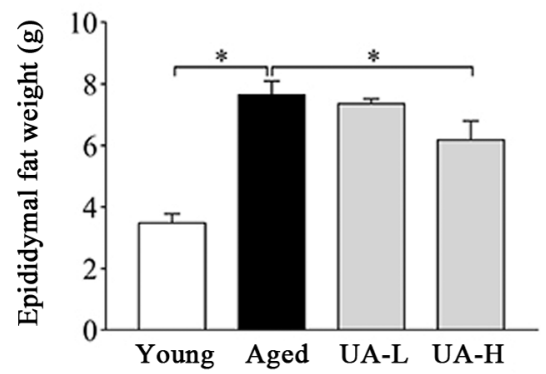

E

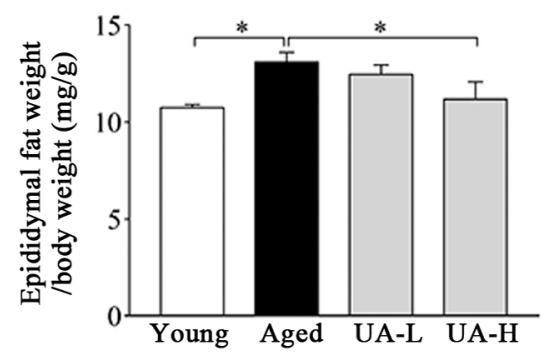

Figure 1. General characteristics of rats included in the present study. (A) Food (chow) intake, (B) body weight, (C) body weight gain, (D) epididymal fat weight and (E) ratio of epididymal fat weight/body weight in rats. Treatment groups were: Young (untreated, $\mathrm{n}=8$ ), aged (untreated, $\mathrm{n}=9$ ), treated with $10 \mathrm{mg} / \mathrm{kg} \mathrm{UA}$ (UA-L, n=8) or $50 \mathrm{mg} / \mathrm{kg}$ UA (UA-H, n=9) for 7 weeks. All animals had free access to a standard diet. ${ }^{*} \mathrm{P}<0.05$ compared with aged group. UA, ursolic acid; UA-L, low ursolic acid; UA-H, high ursolic acid.

incubated in blocking buffer (5\% skimmed milk) for $2 \mathrm{~h}$ at room temperature and immunoblotted with the following primary antibodies for $14 \mathrm{~h}$ at $4^{\circ} \mathrm{C}$ : Rabbit polyclonal anti-IRS-1 antibody (1:1,000; cat. no. TA312859; Origene Technologies, Inc.), rabbit polyclonal anti-phosphorylated(p)-IRS-1 ${ }^{\text {Ser307 }}$ antibody (1:1,000; cat. no. TA325573; Origene Technologies, Inc.), rabbit monoclonal anti-Akt antibody $(1: 1,000$; cat. no. ab179463; Abcam), rabbit monoclonal anti-p-Akt ${ }^{\mathrm{Ser} 473}$ antibody $(1: 1,000$; cat.no.4060; Cell Signaling Technology, Inc.), rabbit polyclonal PI3K antibody (1:6,000; cat. no. 2225185; EMD Millipore), rat monoclonal GLUT4 antibody (1:1,000; cat. no. 10613; Santa Cruz Biotechnology, Inc.), rabbit polyclonal NF- $\kappa \mathrm{B}$ antibody (1:2,000; cat. no. ab16502; Abcam), rabbit polyclonal IL-1 $\beta$ antibody $(1: 1,000$; cat. no. ab9722; Abcam), rat monoclonal IL-6 antibody (1:1,000; cat. no. ab9324; Abcam), and rabbit polyclonal anti-sodium/potassium-transporting ATPase subunit alpha-1 (ATP-1a1) antibody (1:1,000; cat. no. TA326844; Origene Technologies, Inc.). Polyclonal rabbit GAPDH antibody (1:25,000; cat. no. sc-AP0063; Bioworld Technology, Inc.) was used as a loading control to normalize the signal that was obtained. Subsequent to washing (4 times, $10 \mathrm{~min} /$ time) with TBS-0.1\% Tween 20 (TBST), horseradish peroxidase (HRP)-conjugated goat anti-rabbit IgG secondary antibody (1:5,000; cat. no. BA1054; Boster Biological Technology) or goat anti-mouse IgG secondary antibody (1:5,000; cat. no. BA1050; Boster Biological Technology) diluted in phosphate buffered saline were applied to the membrane, followed by incubation for $1.5 \mathrm{~h}$ at room temperature. After washing 4 times with TBST solution for $10 \mathrm{~min}$ each time, the immunoreactive bands were visualized via autoradiography and the density was evaluated using ImageJ v.1.43 (National Institutes of Health). Levels in young rats were arbitrarily assigned a value of 1 .
Statistical analysis. All results are expressed as the mean \pm SEM. At least eight biological replicates were performed. Data were analyzed using StatView software v.5.0 (SAS Institute, Inc.). Data were analyzed by one-way ANOVA followed by the post hoc Student Newman-Keuls and Bonferroni tests to determine differences between groups. $\mathrm{P}<0.05$ was considered to indicate a statistically significant difference.

\section{Results}

Characteristics of rats. The chow intake and body weight of the rats were measured every 2 days. Body weight, food intake, eWAT weight and the ratio of eWAT weight/body weight significantly increased, while body weight gain decreased in the aged group compared with the young normal group (Fig. 1A-E). Treatment with UA-H decreased the weight of eWAT and the ratio of eWAT weight/body weight (Fig. 1D and E); however, UA-L did not (Fig. 1D and E). UA administration did not significantly alter other parameters (Fig. 1A-C). Therefore, the aged rat model has been initially established, and UA may improve the phenomenon of fat accumulation in the epididymis of the aged rats.

Effect of UA on glucose and lipid metabolism disorders in ageing rats. At the end of the experiment, blood was collected from the rats after overnight fasting. As indicated in Fig. 2, aged rats had increased fasting plasma TG, glucose and insulin concentrations, compared with young rats (Fig. 2A-C). Treatment with UA-H partially reversed the increase in plasma TG and insulin concentration (Fig. 2A and C) but had no effect on glucose concentrations (Fig. 2B). In addition, compared with young rats, the HOMA-IR index and Adipo-IR index increased $\sim 2$ fold in 
A



B



C

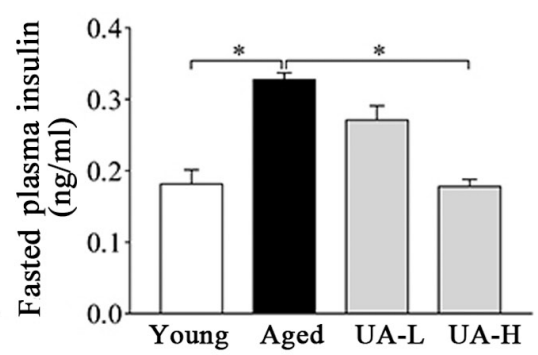

D

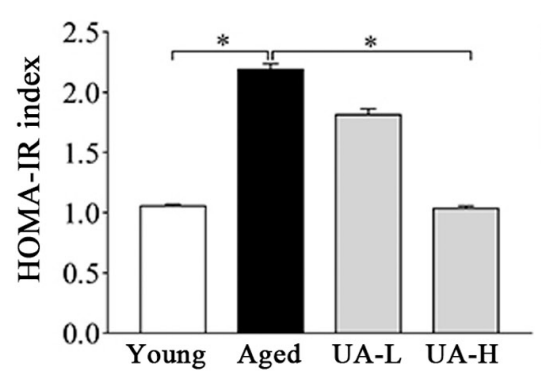

E

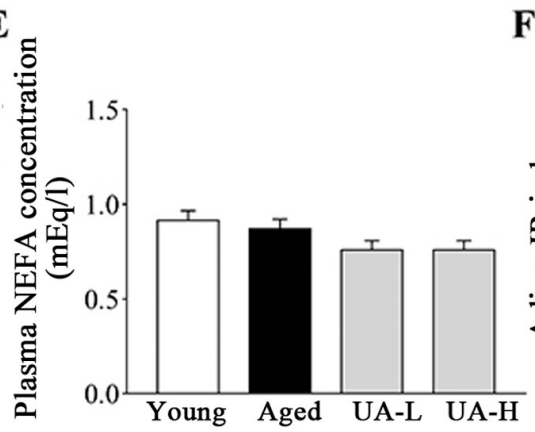

F



Figure 2. Effect of UA on glucose and lipid metabolism disorders in aged rats. (A) Plasma TG concentration, (B) fasted plasma glucose, (C) fasted plasma insulin, (D) HOMA-IR index, (E) plasma NEFA concentration and (F) adipo-IR index in rats. Treatment groups were: Young (untreated, n=8), aged (untreated, $\mathrm{n}=9$ ), treated with $10 \mathrm{mg} / \mathrm{kg}$ UA (UA-L, n=8) or $50 \mathrm{mg} / \mathrm{kg}$ UA (UA-H, $\mathrm{n}=9$ ) for 7 weeks. All animals had free access to a standard diet. * P<0.05 compared with aged group. TG, triglyceride; UA-L, low ursolic acid; UA-H, high ursolic acid; HOMA-IR, the homeostasis model assessment of insulin resistance; NEFA, non-esterified fatty acid; Adipo-IR, adipose tissue insulin resistance.

ageing rats (Fig. 2D and F), indicating increase of systemic and adipose tissue IR with ageing. Oral administration of UA-H reversed the increase in the HOMA-IR index and Adipo-IR index (Fig. 2D and F), but the UA-L group demonstrated minor changes (Fig. 2D and F). Plasma NEFA had no significant differences between all the rat groups tested (Fig. 2E). Taken together, these results suggested that treatment with UA-H improves metabolic effects compared with UA-L treatment.

Effect of UA on the IRS-1/PI3K/Akt signaling pathway in eWAT of ageing rats. Subsequently, the effect of UA on insulin signaling pathways was further investigated in the UA-H group. Western blotting was used to investigate the adipose tissue proteins of the epididymis of rats in each group. The expression of IRS-1, p-IRS-1 (ser307) and p-Akt (ser473), and the ratio of p-Akt (ser473) to Akt were all significantly decreased in the eWAT of ageing rats, compared with young normal rats (Fig. 3A). Treatment with UA-H significantly reversed all these changes, except for the ratio of p-IRS-1 (ser307) to IRS-1 protein (Fig. 3A). In addition, the expression of PI3K and Akt did not differ between the young, aged and UA-H groups (Fig. 3A and B). The aforementioned results indicated that UA ameliorated eWAT IR in ageing rats, which was associated with the Akt signaling pathway.

Effect of UA on GLUT4 translocation in eWAT of ageing rats. Membrane proteins and total proteins were extracted and detected via western blotting. GLUT4 total protein expression was significantly decreased in eWAT of aged rats compared with the young normal group (Fig. 4A). Treatment with UA-H significantly upregulated GLUT4 expression in the aged rats
(Fig. 4A). Due to GLUT4 primarily exerting its effects in the plasma membrane, the impact of UA on GLUT4 on the plasma membrane and cytoplasmic Glut4 in eWAT of ageing rats was evaluated. As expected, UA-H significantly inhibited the decrease of GLUT4 on the plasma membrane and cytoplasmic Glut4 in the adipocytes of ageing rats (Fig. 4B and C). These results indicated that ageing may result in impaired glucose transport and UA may regulate the translocation of GLUT4 to facilitate glucose intake in eWAT.

Effect of UA on inflammation in eWAT of ageing rats. Western blotting results revealed that the protein expression of $\mathrm{NF}-\kappa \mathrm{B}$ and proinflammatory cytokines IL- 6 and IL-1 $\beta$ were higher in aged rats compared with young normal rats (Fig. 5A-D). Treatment with UA-H significantly decreased these factors (Fig. 5A-D) indicating that UA may alleviate age-related adipose tissue inflammatory.

\section{Discussion}

During ageing, the body's metabolic changes are closely associated with several prevalent medical conditions, such as obesity, IR, type 2 diabetes, cardiovascular diseases, such as hyperlipidemia and coronary atherosclerosis, and neurodegenerative disorders, such as Alzheimer's disease and Parkinson's disease (30). Of these, IR is the common pathophysiological basis and risk factor of multiple metabolic diseases, which is the premise of the present study $(31,32)$. HOMA-IR is a simple and useful indicator in the assessment of IR in epidemiological studies (33) and is widely used for the determination of glucose and insulin levels to reflect systemic IR (34). Adipo-IR is often 
A



B



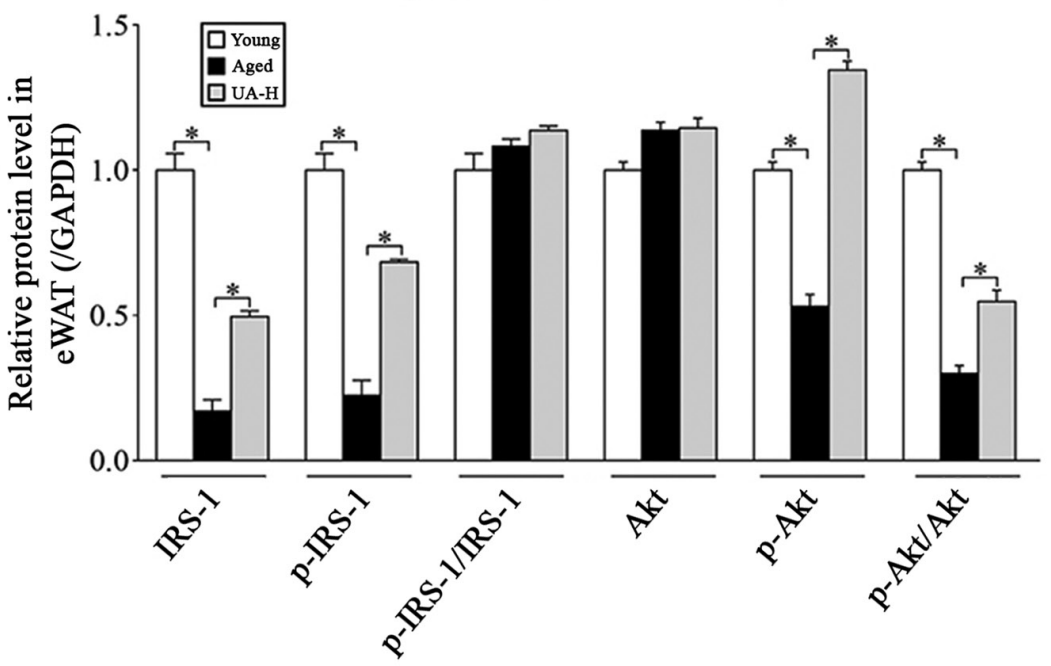

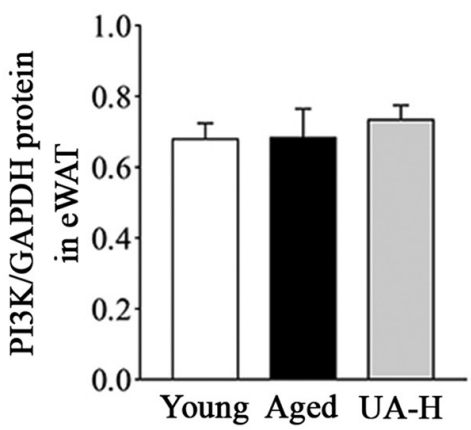

Figure 3. Effect of UA on IRS-1/PI3K/Akt signaling pathway in eWAT of aged rats. (A) Expression of IRS-1, p-IRS-1 (Ser307), p-IRS-1 (Ser307)/IRS-1, Akt, p-Akt (Ser473) and p-Akt (Ser473)/Akt protein levels. (B) PI3K protein levels. Treatment groups were: Young (untreated, $n=8$ ), aged (untreated, $n=9$ ), treated with $10 \mathrm{mg} / \mathrm{kg}$ UA (UA-L, $\mathrm{n}=8$ ) or $50 \mathrm{mg} / \mathrm{kg}$ UA (UA-H, $\mathrm{n}=9$ ) for 7 weeks. All animals had free access to a standard diet. "P<0.05 compared with aged group. UA-H, high ursolic acid; eWAT, epididymis white adipose tissue; IRS-1, insulin receptor substrate-1; p, phosphorylated; Akt, protein kinase B; PI3K, phosphatidylinositol 3-kinase.

A
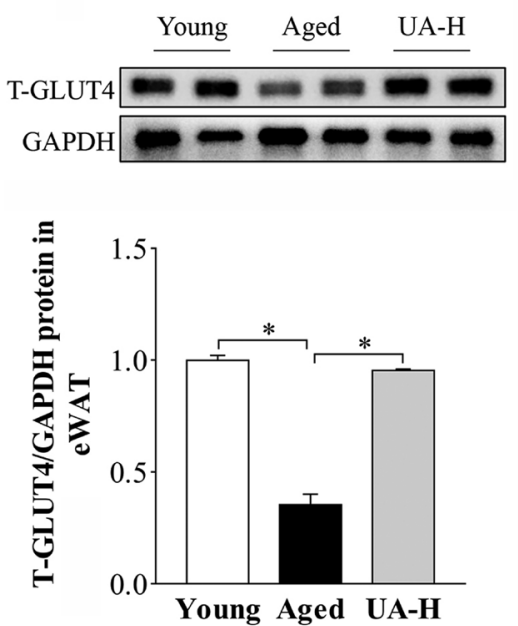

B
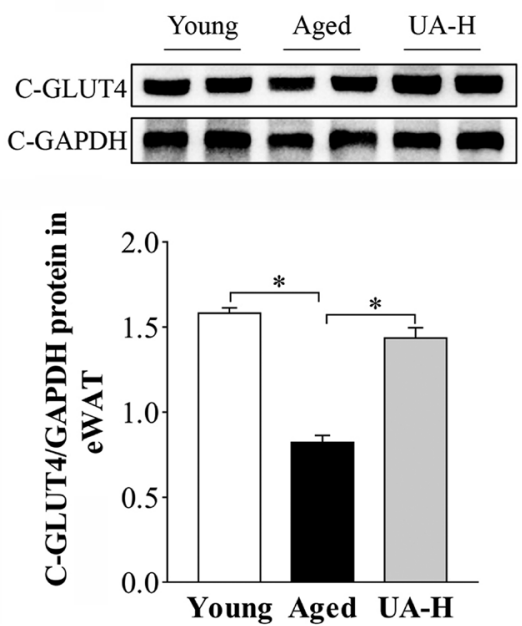

C
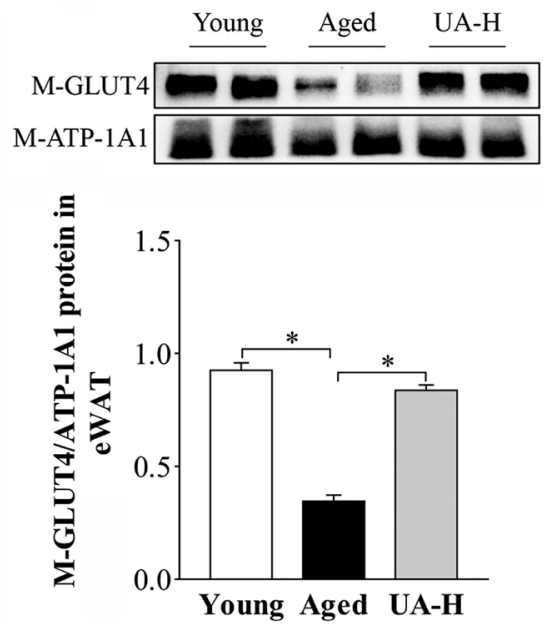

Figure 4. Effect of UA on Glut4 translocation in eWAT of aged rats. Protein expression of (A) T-Glut4 protein levels, (B) C-Glut4 protein levels and (C) M-Glut4 protein levels in eWAT of rats. Treatment groups were: Young (untreated, $\mathrm{n}=8$ ), aged (untreated, $\mathrm{n}=9$ ), treated with $10 \mathrm{mg} / \mathrm{kg} \mathrm{UA}(\mathrm{UA}-\mathrm{L}, \mathrm{n}=8$ ) or $50 \mathrm{mg} / \mathrm{kg} \mathrm{UA}$ (UA-H, n=9) for 7 weeks. All animals had free access to a standard diet. ${ }^{*} \mathrm{P}<0.05$ compared with aged group. UA-H, high ursolic acid; eWAT, epididymis white adipose tissue; T-Glut4, Glut4 total protein; C-Glut4, cytoplasmic Glut4; M-Glut4, Glut4 on the plasma membrane; GLUT4, glucose transporter 4.

used as an effective indicator to measure insulin sensitivity in adipose tissue (35). It has been reported that adipose tissue is a tissue type in which inflammation and IR are established particularly early during ageing (36). Epididymal fat is a representative visceral adipose tissue, which influences metabolic processes due to its active secretion of cytokines (visfatin, adiponectin) and chemokines (C-C motif chemokine 2, IL-8), and is widely used in metabolic studies (37). In the present study, compared with young rats, the aged rats had significantly higher eWAT weight, plasma glucose, insulin, TG concentration, HOMA-IR index and Adipo-IR, which indicated ageing-associated adipose tissue IR. It 
A

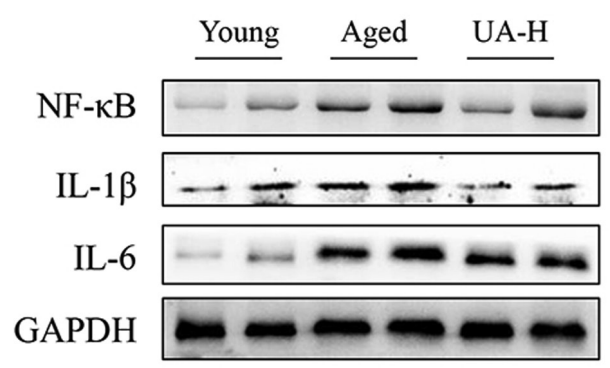

C

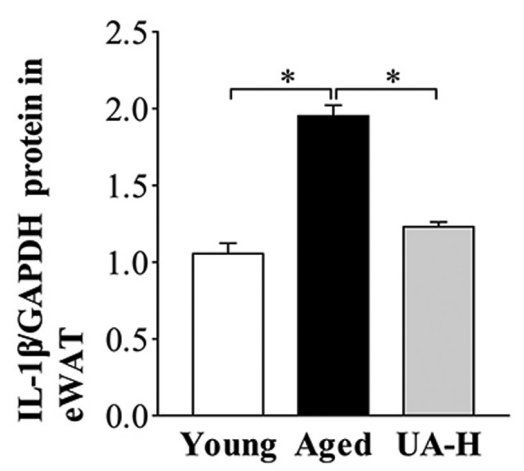

B

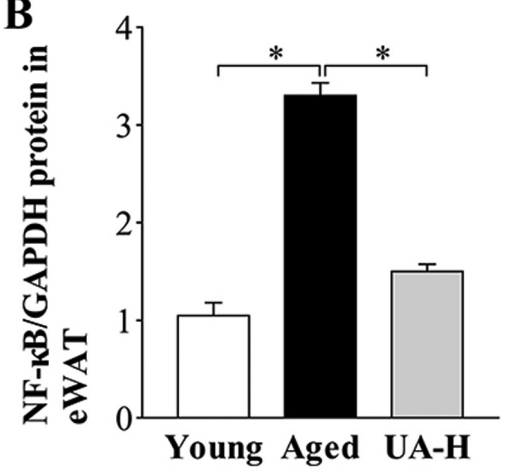

D

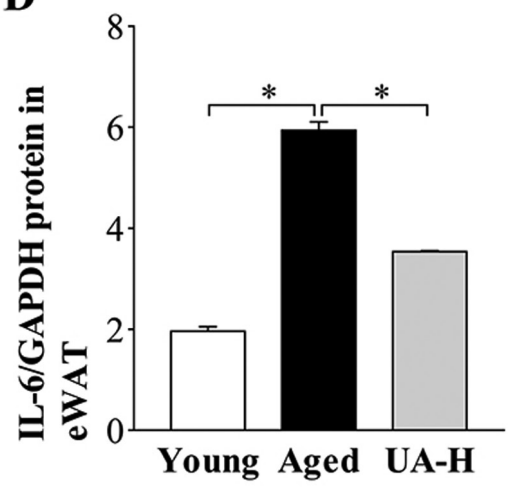

Figure 5. Effect of UA on inflammatory markers in eWAT of aged rats. (A) Representative images of western blot analysis of NF- $\kappa$ B, IL-6 and IL-1 $\beta$ in eWAT. (B) NF- $\mathrm{B}$ (C) IL-1 $\beta$ and (D) IL-6 protein expression levels in eWAT of rats. Treatment groups were: Young (untreated, $\mathrm{n}=8$ ), aged (untreated, $\mathrm{n}=9$ ), treated with $10 \mathrm{mg} / \mathrm{kg}$ UA (UA-L, n=8) or $50 \mathrm{mg} / \mathrm{kg}$ UA (UA-H, $\mathrm{n}=9$ ) for 7 weeks. All animals had free access to a standard diet. * $<<0.05$ compared with aged group. UA-H, high ursolic acid; eWAT, epididymis white adipose tissue; NF- $\kappa \mathrm{B}$, nuclear factor $\kappa \mathrm{B}$; IL, interleukin.

has been reported that UA regulates the ageing process via enhancement of metabolic sensor protein levels (including sirtuin 1, sirtuin 6 and proliferator-activated receptor gamma coactivator-1 $\beta$ ) (38). Previous studies have demonstrated that dietary UA improves health and life span in male Drosophila melanogaster (39), and exerts an antiaging effect by promoting mice skeletal muscle rejuvenation in C57BL/6 (40). Notably, in the present study, UA treatment significantly decreased the aforementioned parameters (eWAT weight, plasma glucose, insulin, TG concentration, HOMA-IR index and Adipo-IR) in aged rats. The findings of the present study suggested that UA may improve ageing-related adipose tissue IR; however, the possible mechanism for this needs further verification.

The key mechanism of IR is hypothesized to be the disturbance of the insulin signaling pathway (41). In addition, the PI3K/Akt signaling pathway serves an important role in the metabolic function of insulin, which regulates the absorption and metabolism of glucose $(42,43)$. In addition, in adipocytes, IR has been attributed to post-insulin receptor defects, making IRS-1 and Akt appropriate candidates to study insulin pathway alterations (44). Ser307 in IRS-1 has been revealed as a molecular indicator of IR, when the serine residues of IRS-1 are phosphorylated in response to stimuli (TNF- $\alpha$, insulin) (45). There is a progressive decline of insulin-stimulated phosphorylation associated with ageing, such as insulin-induced IR and IRS-1 phosphorylation (46). Similarly, the findings of the present study demonstrated that the expression of IRS-1 and p-IRS-1(ser307) was inhibited in eWAT of ageing rats compared with the young normal group. In the present study, oral administration of UA reversed these changes, but it did not alter the ratio of p-IRS-1 (ser307)/IRS-1. IRS-1 couples insulin receptor signaling to a PI3K-dependent pathway and activated PI3K regulates insulin metabolism primarily via IRS-1 (47). Other molecules downstream of PI3K, such as Akt/mTOR and Akt/NF- $\kappa \mathrm{B}$, are also altered (46). Akt is a key protein located downstream of PI3K, which stimulates the GLUT4 transmembrane receptor to transport glucose, accelerating glycometabolism (48). Several studies have suggested that ageing deteriorates both systemic insulin sensitivity in aged ovariectomized female rats $(49,50)$, and GLUT4 total expression levels in aged female rats $(51,52)$ in the cerebral cortex. In addition, in adipose tissue of transgenic mice, insulin-stimulated transport of glucose was primarily mediated by GLUT4 (51). Under the stimulation of insulin, GLUT4 promoted the translocation of glucose to the cell membrane, promoting glucose uptake and metabolism in 3T3-L1 adipocytes (53). Hence, the decrease in GLUT4 activity associated with ageing may result in a barrier to glucose uptake from fat and result in a decrease in insulin sensitivity. Similarly, the findings of the present study revealed that the protein expression of IRS-1 (ser307)/p-IRS-1, p-Akt (Ser473)/Akt, and both total GLUT4 and cellular membrane GLUT4 were inhibited in eWAT of aged rats compared with the young normal group, indicating the age-related impairment of the IRS-1-PI3K-Akt/GLUT4 pathway. In the present study, although UA had no effect on p-IRS-1 (ser307)/IRS-1, PI3K and total Akt, it significantly reversed the decrease in the ratio of p-Akt (Ser473)/Akt and the 
level of GLUT4 on the plasma membrane of adipocytes. The findings of the present study suggested that UA may improve adipose tissue insulin sensitivity to facilitate glucose uptake and metabolism, which is associated with Akt activation and GLUT4 membrane translocation.

Although, all tissues exhibit signs of inflammation and IR with ageing, epididymal fat is the first to develop age-associated signs of inflammation and IR of the white fat tissues (35). Chronic systemic inflammation without apparent infection in elderly humans is often referred to as the 'inflammageing' phenotype and is primarily characterized by elevated levels of pro-inflammatory cytokines (IL-6, TNF- $\alpha$ ) (54-59). The present study demonstrated that the levels of pro-inflammatory cytokines IL- 6 and IL- $1 \beta$ are significantly elevated in the eWAT of ageing rats compared with the young normal group. Previous studies have demonstrated that $\mathrm{NF}-\kappa \mathrm{B}$ serves an important role in obesity-related inflammation, by activating the production of pro-inflammatory factors, such as activator protein- 1 , TNF- $\alpha$ and IL-6 (60-63). In addition, it has been reported that in mice with diabetes-induced nephropathy, UA alleviated the levels of pro-inflammatory factors TNF- $\alpha$, IL-1 $\beta$, IL-6 and IL-8 (64). Concordantly, the present study revealed that UA significantly decreased expression levels of pro-inflammatory factors, such as IL- 6 and IL- $1 \beta$ in the eWAT of aged rats compared with aged group, which may be associated with UA regulation of $\mathrm{NF}-\kappa \mathrm{B}$ signaling. It has been demonstrated that the insulin receptor signaling pathway and inflammatory cytokines (TNF- $\alpha$, IL-1 $\beta$, IL-6) ross-talk with each other (65). Hence, the amelioration of insulin sensitivity induced by UA may be associated with inhibiting proinflammatory factor release in adipose tissue of aged rats. The limitation of the present study is that in vitro experiments were not performed. Future work will verify the current conclusions through in vitro cell experiments.

In conclusion, the present study demonstrated that UA had favorable effects on hyperinsulinemia, hypertriglyceridemia and pro-inflammatory cytokine expression in eWAT of aged rats, indicating that UA may improve age-related IR and inflammation in adipose tissue. The findings of the present study indicated that UA has potential as a therapeutic agent for IR and metabolic syndromes associated with ageing, and provides a basis for the development of effective and safe drugs or functional foods for the prevention and treatment of metabolic diseases, including hypertension, coronary heart disease and obesity.

\section{Acknowledgements}

The authors would like to thank Dr Chun-Li Li (Institute of Life Sciences, Chongqing Medical University, Chongqing, China) and Dr Da-Zhi Ke (The Second Affiliated Hospital of Chongqing Medical University, Chongqing, China) for their critical revisions and constructive comments on the content of the present study.

\section{Funding}

The present study was supported by the National Natural Science Foundation of China (grant nos. 81374033 and 81673659), Chongqing Postgraduate Research and Innovation Project Fund (grant no. CYS18210), Project of Chongqing Education Commission (grant. no. KJ1600233) and Cultivation Fund of Chongqing medical university (grant no. X12100).

\section{Availability of data and materials}

The datasets used and/or analyzed during the current study are available from the corresponding author on reasonable request.

\section{Authors' contributions}

TZW, GWZ and JWW conceived and designed the experiments of the current study. TZW, LY, CLY, HFL, YL, ZWC, YQJ and JZ performed the experiments. TZW, CLY, HFL and GWZ analyzed the data. JY drafted the work and reviewed the research design. DZK, CLL and JY revised the manuscript critically for important intellectual content. JWW provided reagents, materials and analysis tools and finally approved the version to be released. TZW wrote the manuscript. GWZ and JWW were responsible for reviewing the manuscript for important intellectual content. TZW, GWZ and JWW confirm the authenticity of all the raw data. All authors have read and approved the final manuscript.

\section{Ethics approval and consent to participate}

All animal experiments were performed in accordance with the institutional and national guidelines and regulations, and approved by the Chongqing Medical University Animal Care and Use Committee.

\section{Patient consent for publication}

Not applicable.

\section{Competing interests}

The authors declare that they have no competing interests.

\section{References}

1. Bai Y, Bian F, Zhang L and Cao Y: The impact of social support on the health of the rural elderly in China. Int J Environ Res Public Health 17: 2004, 2020.

2. Evans JL and Goldfine ID: Aging and insulin resistance: Just say iNOS. Diabetes 62: 346-348, 2013.

3. Hanson RL, Imperatore G, Bennett PH and Knowler WC Components of the 'metabolic syndrome' and incidence of type 2 diabetes. Diabetes 51: 3120-3127, 2002.

4. Joannides CN, Mangiafico SP, Waters MF, Lamont BJ and Andrikopoulos S: Dapagliflozin improves insulin resistance and glucose intolerance in a novel transgenic rat model of chronic glucose overproduction and glucose toxicity. Diabetes Obes Metab 19: 1135-1146, 2017

5. Jeong $\mathrm{JH}$ and Kang EB: Effects of treadmill exercise on PI3K/ AKT/GSK-3 $\beta$ pathway and tau protein in high-fat diet-fed rats. J Exerc Nutrition Biochem 22: 9-14, 2018.

6. Li Y, Wang J, Gu T, Yamahara J and Li Y: Oleanolic acid supplement attenuates liquid fructose-induced adipose tissue insulin resistance through the insulin receptor substrate-1/phosphatidylinositol 3-kinase/Akt signaling pathway in rats. Toxicol Appl Pharmacol 277: 155-163, 2014.

7. Luan G, Li G, Ma X, Jin Y, Hu N, Li J, Wang Z and Wang H: Dexamethasone-induced mitochondrial dysfunction and insulin resistance-study in 3T3-L1 adipocytes and mitochondria isolated from mouse liver. Molecules 24: 1982, 2019. 
8. Wang F, Han L, Qin RR, Zhang YY, Wang D, Wang ZH, Tang MX, Zhang Y, Zhong $M$ and Zhang W: Overexpressing STAMP2 attenuates adipose tissue angiogenesis and insulin resistance in diabetic ApoE ${ }^{-/} / \mathrm{LDLR}^{-/}$mouse via a PPAR $\gamma / \mathrm{CD} 36$ pathway. J Cell Mol Med 21: 3298-3308, 2017.

9. Lee BC and Lee J: Cellular and molecular players in adipose tissue inflammation in the development of obesity-induced insulin resistance. Biochim Biophys Acta 1842: 446-462, 2014.

10. Cui X, Qian DW, Jiang S, Shang EX, Zhu ZH and Duan JA Scutellariae radix and coptidis rhizoma improve glucose and lipid metabolism in T2DM rats via regulation of the metabolic profiling and MAPK/PI3K/Akt signaling pathway. Int J Mol Sci 19: 3634, 2018.

11. Cusi K, Maezono K, Osman A, Pendergrass M, Patti ME, Pratipanawatr T, DeFronzo RA, Kahn CR and Mandarino LJ: Insulin resistance differentially affects the PI 3-kinase- and MAP kinase-mediated signaling in human muscle. J Clin Invest 105 : 311-320, 2000.

12. Brachmann SM, Ueki K, Engelman JA, Kahn RC and Cantley LC: Phosphoinositide 3-kinase catalytic subunit deletion and regulatory subunit deletion have opposite effects on insulin sensitivity in mice. Mol Cell Biol 25: 1596-1607, 2005.

13. Kanai F, Ito K, Todaka M, Hayashi H, Kamohara S, Ishii K, Okada T, Hazeki O, Ui M and Ebina Y: Insulin-stimulated GLUT4 translocation is relevant to the phosphorylation of IRS-1 and the activity of PI3-kinase. Biochem Biophys Res Commun 195 762-768, 1993.

14. Cantley LC: The phosphoinositide 3-kinase pathway. Science 296 $1655-1657,2002$.

15. Kohn AD, Summers SA, Birnbaum MJ and Roth RA: Expression of a constitutively active Akt Ser/Thr kinase in 3T3-L1 adipocytes stimulates glucose uptake and glucose transporter 4 translocation. J Biol Chem 271: 31372-31378, 1996.

16. Gandhi GR, Stalin A, Balakrishna K, Ignacimuthu S, Paulraj MG and Vishal R: Insulin sensitization via partial agonism of PPAR $\gamma$ and glucose uptake through translocation and activation of GLUT4 in PI3K/p-Akt signaling pathway by embelin in type 2 diabetic rats. Biochim Biophys Acta 1830: 2243-2255, 2013.

17. Liu J: Oleanolic acid and ursolic acid: Research perspectives. J Ethnopharmacol 100: 92-94, 2005

18. Ikeda Y, Murakami A and Ohigashi H: Ursolic acid: An anti- and pro-inflammatory triterpenoid. Mol Nutr Food Res 52: 26-42, 2008 .

19. Li S, Liao X, Meng F, Wang Y, Sun Z, Guo F, Li X, Meng M, Li Y and Sun C: Therapeutic role of ursolic acid on ameliorating hepatic steatosis and improving metabolic disorders in high-fat diet-induced non-alcoholic fatty liver disease rats. PLoS One 9: e86724, 2014

20. Chu X, He X, Shi ZP, Li CJ, Guo FC, Li ST, Li Y, Na LX and Sun $\mathrm{CH}$ : Ursolic acid increases energy expenditure through enhancing free fatty acid uptake and $\beta$-oxidation via an UCP3 AMPK-dependent pathway in skeletal muscle. Mol Nutr Food Res 59: 1491-1503, 2015.

21. Ma P, Yao L, Lin X, Gu T, Rong X, Batey R, Yamahara J, Wang $\mathrm{J}$ and $\mathrm{Li} \mathrm{Y}$ : A mixture of apple pomace and rosemary extract improves fructose consumption-induced insulin resistance in rats: Modulation of sarcolemmal CD36 and glucose transporter-4. Am J Transl Res 8: 3791-3801, 2016.

22. Jäger S, Trojan H, Kopp T, Laszczyk MN and Scheffler A Pentacyclic triterpene distribution in various plants-rich sources for a new group of multi-potent plant extracts. Molecules 14: 2016-2031, 2009.

23. Jia YY, Kim S, Kim J, Kim B, Wu CY, Lee JH, Jun HJ, Kim N, Lee D and Lee SJ: Ursolic acid improves lipid and glucose metabolism in high-fat-fed C57BL/6J mice by activating peroxisome proliferator-activated receptor alpha and hepatic autophagy. Mol Nutr Food Res 59: 344-354, 2015.

24. Gastaldelli A, Harrison SA, Belfort-Aguilar R, Hardies LJ, Balas B, Schenker S and Cusi K: Importance of changes in adipose tissue insulin resistance to histological response during thiazolidinedione treatment of patients with nonalcoholic steatohepatitis. Hepatology 50: 1087-1093, 2009.

25. Bugianesi E, Gastaldelli A, Vanni E, Gambino R, Cassader M, Baldi S, Ponti V, Pagano G, Ferrannini E and Rizzetto M: Insulin resistance in non-diabetic patients with non-alcoholic fatty liver disease: Sites and mechanisms. Diabetologia 48: 634-642, 2005.

26. Gastaldelli A, Cusi K, Pettiti M, Hardies J, Miyazaki Y, Berria R, Buzzigoli E, Sironi AM, Cersosimo E, Ferrannini E and Defronzo RA: Relationship between hepatic/visceral fat and hepatic insulin resistance in nondiabetic and type 2 diabetic subjects. Gastroenterology 133: 496-506, 2007.
27. Lomonaco R, Ortiz-Lopez C, Orsak B, Webb A, Hardies J, Darland C, Finch J, Gastaldelli A, Harrison S, Tio F and Cusi K: Effect of adipose tissue insulin resistance on metabolic parameters and liver histology in obese patients with nonalcoholic fatty liver disease. Hepatology 55: 1389-1397, 2012.

28. Armstrong MJ, Hazlehurst JM, Hull D, Guo K, Borrows S, Yu J, Gough SC, Newsome PN and Tomlinson JW: Abdominal subcutaneous adipose tissue insulin resistance and lipolysis in patients with non-alcoholic steatohepatitis. Diabetes Obes Metab 16: 651-660, 2014.

29. Bell LN, Wang J, Muralidharan S, Chalasani S, Fullenkamp AM, Wilson LA, Sanyal AJ, Kowdley KV, Neuschwander-Tetri BA, Brunt EM, et al: Relationship between adipose tissue insulin resistance and liver histology in nonalcoholic steatohepatitis: A pioglitazone versus vitamin $E$ versus placebo for the treatment of nondiabetic patients with nonalcoholic steatohepatitis trial follow-up study. Hepatology 56: 1311-1318, 2012.

30. Dagdeviren S, Jung DY, Friedline RH, Noh HL, Kim JH, Patel PR, Tsitsilianos N, Inashima K, Tran DA, Hu X, et al: IL-10 prevents ageing-associated inflammation and insulin resistance in skeletal muscle. FASEB J 31: 701-710, 2017.

31. Chiyanika C, Chan DFY, Hui SCN, So HK, Deng M, Yeung DKW, Nelson EAS and Chu WCW: The relationship between pancreas steatosis and the risk of metabolic syndrome and insulin resistance in Chinese adolescents with concurrent obesity and non-alcoholic fatty liver disease. Pediatr Obes 15: e12653, 2020.

32. Yan H, Li T, Wang Y, Li H, Xu J and Lu X: Insulin-like growth factor binding protein 7 accelerates hepatic steatosis and insulin resistance in non-alcoholic fatty liver disease. Clin Exp Pharmacol Physiol 46: 1101-1110, 2019.

33. Antunes LC, Elkfury JL, Jornada MN, Foletto KC and Bertoluci MC: Validation of HOMA-IR in a model of insulin-resistance induced by a high-fat diet in Wistar rats. Arch Endocrinol Metab 60: 138-142, 2016.

34. Matthews DR, Hosker JP, Rudenski AS, Naylor BA, Treacher DF and Turner RC: Homeostasis model assessment: Insulin resistance and beta-cell function from fasting plasma glucose and insulin concentrations in man. Diabetologia 28: 412-419, 1985.

35. Søndergaard E, Espinosa De Ycaza AE, Morgan-Bathke M and Jensen MD: How to measure adipose tissue insulin sensitivity. J Clin Endocrinol Metab 102: 1193-1199, 2017.

36. Sierra Rojas JX, García-San Frutos M, Horrillo D, Lauzurica N, Oliveros E, Carrascosa JM, Fernández-Agulló T and Ros M: Differential development of inflammation and insulin resistance in different adipose tissue depots along ageing in Wistar rats: Effects of caloric restriction. J Gerontol A Biol Sci Med Sci 71: 310-322, 2016.

37. Altintas MM, Azad A, Nayer B, Contreras G, Zaias J, Faul C, Reiser J and Nayer A: Mast cells, macrophages, and crown-like structures distinguish subcutaneous from visceral fat in mice. J Lipid Res 52: 480-488, 2011.

38. Bahrami SA and Bakhtiari N: Ursolic acid regulates ageing process through enhancing of metabolic sensor proteins level. Biomed Pharmacother 82: 8-14, 2016.

39. Staats S, Wagner AE, Lüersen K, Künstner A, Meyer T, Kahns AK, Derer S, Graspeuntner S, Rupp J, Busch H, et al: Dietary ursolic acid improves health span and life span in male Drosophila melanogaster. Biofactors 45: 169-186, 2019.

40. Bakhtiari N, Hosseinkhani S, Tashakor A and Hemmati R: Ursolic acid ameliorates ageing-metabolic phenotype through promoting of skeletal muscle rejuvenation. Med Hypotheses 85: $1-6,2015$.

41. Zhou X, Li JQ, Wei LJ, He MZ, Jia J, Zhang JY, Wang SS and Feng L: Silencing of DsbA-L gene impairs the PPAR $\gamma$ agonist function of improving insulin resistance in a high-glucose cell model. J Zhejiang Univ Sci B 21: 990-998, 2020.

42. Jiang S, Ren D, Li J, Yuan G, Li H, Xu G, Han X, Du P and An L: Effects of compound $\mathrm{K}$ on hyperglycemia and insulin resistance in rats with type 2 diabetes mellitus. Fitoterapia 95: 58-64, 2014.

43. Vareda PM, Saldanha LL, Camaforte NA, Violato NM, Dokkedal AL and Bosqueiro JR: Myrcia bella leaf extract presents hypoglycemic activity via PI3k/Akt insulin signaling pathway. Evid Based Complement Alternat Med 2014: 543606, 2014.

44. Ormazabal P, Scazzocchio B, Varì R, Santangelo C D'Archivio M, Silecchia G, Iacovelli A, Giovannini C and Masella R: Effect of protocatechuic acid on insulin responsiveness and inflammation in visceral adipose tissue from obese individuals: Possible role for PTP1B. Int J Obes (Lond) 42: 2012-2021, 2018 
45. Aguirre V, Werner ED, Giraud J, Lee YH, Shoelson SE and White MF: Phosphorylation of Ser307 in insulin receptor substrate-1 blocks interactions with the insulin receptor and inhibits insulin action. J Biol Chem 277: 1531-1537, 2002.

46. García-San Frutos M, Fernández-Agulló T, De Solís AJ, Andrés A, Arribas C, Carrascosa JM and Ros M: Impaired central insulin response in aged Wistar rats: Role of adiposity. Endocrinology 148: 5238-5247, 2007.

47. Aziz AUR, Farid S, Qin K, Wang H and Liu B: Regulation of insulin resistance and glucose metabolism by interaction of PIM kinases and insulin receptor substrates. Arch Physiol Biochem 126: 129-138, 2020.

48. Gandhi GR, Jothi G, Antony PJ, Balakrishna K, Paulraj MG, Ignacimuthu S, Stalin A and Al-Dhabi NA: Gallic acid attenuates high-fat diet fed-streptozotocin-induced insulin resistance via partial agonism of PPAR $\gamma$ in experimental type 2 diabetic rats and enhances glucose uptake through translocation and activation of GLUT4 in PI3K/p-Akt signaling pathway. Eur J Pharmacol 745 201-216, 2014

49. Alonso A, González-Pardo H, Garrido P, Conejo NM, Llaneza P, Díaz F, Del Rey CG and González C: Acute effects of $17 \beta$-estradiol and genistein on insulin sensitivity and spatial memory in aged ovariectomized female rats. Age (Dordr) 32: 421-434, 2010.

50. Alonso A,Fernández R,Moreno M,Ordóñez P,González-Pardo H Conejo NM, Díaz F and González C: Positive effects of 17beta-estradiol on insulin sensitivity in aged ovariectomized female rats. J Gerontol A Biol Sci Med Sci 61: 419-426, 2006.

51. Shaohui H and Michael PC: The GLUT4 glucose transporter. Cell Metab 5: 237-252, 2007

52. Morán J, Garrido P, Alonso A, Cabello E and González C: $17 \beta$-estradiol and genistein acute treatments improve some cerebral cortex homeostasis aspects deteriorated by ageing in female rats. Exp Gerontol 48: 414-421, 2013.

53. Lu Y, Ma X, Kong Q, Xu Y, Hu J, Wang F, Qin W, Wang L and Xiong W: Novel dual-color drug screening model for GLUT4 translocation in adipocytes. Mol Cell Probes 43: 6-12, 2019.

54. Day MJ: Ageing, immunosenescence and inflammageing in the dog and cat. J Comp Pathol 142 (Suppl 1): S60-S69, 2010.
55. Franceschi C, Bonafè M, Valensin S, Olivieri F, De Luca M, Ottaviani E and De Benedictis G: Inflamm-ageing. An evolutionary perspective on immunosenescence. Ann N Y Acad Sci 908: 244-254, 2000

56. Ahmad A, Banerjee S, Wang Z, Kong D, Majumdar AP and Sarkar FH: Ageing and inflammation: Etiological culprits of cancer. Curr Ageing Sci 2: 174-186, 2009.

57. Fulop T, Larbi A, Kotb R, de Angelis F and Pawelec G: Ageing, immunity, and cancer. Discov Med 11: 537-550, 2011.

58. Khatami M: Inflammation, ageing, and cancer: Tumoricidal versus tumorigenesis of immunity: A common denominator mapping chronic diseases. Cell Biochem Biophys 55: 55-79, 2009.

59. Morrisette-Thomas V,Cohen AA,Fülöp T,RiescoÉ,Legault V,LiQ, Milot E, Dusseault-Bélanger F and Ferrucci L: Inflamm-aging does not simply reflect increases in pro-inflammatory markers. Mech Ageing Dev 139: 49-57, 2014.

60. Hirosumi J, Tuncman G, Chang L, Görgün CZ, Uysal KT, Maeda K, Karin M and Hotamisligil GS: A central role for JNK in obesity and insulin resistance. Nature 420: 333-336, 2002.

61. Maekawa T, Jin W and Ishii S: The role of ATF-2 family transcription factors in adipocyte differentiation: Antiobesity effects of p38 inhibitors. Mol Cell Biol 30: 613-625, 2010

62. Yuan M, Konstantopoulos N, Lee J, Hansen L, Li ZW, Karin M and Shoelson SE: Reversal of obesity- and diet-induced insulin resistance with salicylates or targeted disruption of Ikkbeta. Science 293: 1673-1677, 2001.

63. Arkan MC, Hevener AL, Greten FR, Maeda S, Li ZW, Long JM, Wynshaw-Boris A, Poli G, Olefsky J and Karin M: IKK-beta links inflammation to obesity-induced insulin resistance. Nat Med 11: 191-198, 2005.

64. Li J, Li N, Yan S, Liu M, Sun B, Lu Y and Shao Y: Ursolic acid alleviates inflammation and against diabetes-induced nephropathy through TLR4-mediated inflammatory pathway. Mol Med Rep 18: 4675-4681, 2018.

65. Liu S, Li X, Wu Y, Duan R, Zhang J, Du F, Zhang Q, Li Y and $\mathrm{Li}$ N: Effects of vaspin on pancreatic $\beta$ cell secretion via PI3K/ Akt and NF-kB signaling pathways. PLoS One 12: e0189722, 2017. 